\title{
Apport de la modélisation de la stabilité du manteau neigeux dans la chaîne de modélisation des avalanches de neige sèches.
}

\author{
Contribution of the snow cover stability modeling \\ to the avalanche phenomenon analysis \\ par Lionel Vidal, Transmontagne et Cemagref, \\ Mohamed Naaim et Florence Bouvet-Naaim, Cemagref-ETNA
}

This article deals with the stability of the snow cover in a simplified case of a two layer stratification including a cohesive layer laying on a weak layer. A parametric study at a macroscopic scale is presented, as well as a study of the coupling mechanisms between these two layers. The results are presented and discussed. This study is replaced within the framework of a chain of modeling of the avalanche phenomenon chronology dealing with the problem since the production conditions to the flow phase.

$$
\begin{aligned}
& \text { NOTATION : } \\
& -e_{p} \text { : épaisseur de la plaque. } \\
& -e_{s} \text { : épaisseur de la couche fragile. } \\
& -\rho: \text { densité de la plaque. } \\
& -C: \text { cohésion de la couche. } \\
& -\phi: \text { angle de frottement interne de la couche. }
\end{aligned}
$$

\section{D LA MODÉLISATION IMBRIQUÉE}

Les modèles de transport de neige par le vent, de stabilité du manteau neigeux et d'écoulement des avalanches traitent de manière indépendante les différentes phases de l'avalanche. Le modèle de transport de neige par le vent permet de déterminer la distribution spatiale de la neige dans la zone de départ qui constitue l'entrée du modèle de stabilité qui lui-même fournit une condition initiale au modèle d'écoulement.

En France les données nivo-météorologiques des zones de montagnes sont acquises, stockées et gérées par Météo France. L'échelle d'espace adoptée pour ces données est le massif, dont la superficie est de l'ordre de $1000 \mathrm{~km}^{2}$. Les avalanches les plus importantes en termes de dégâts et d'extension se produisent à la suite de fortes chutes de neige par temps froid et accompagnées de vent. Dans ce cas la neige est fraîche et sèche. Il suffit alors d'un vent de $4 \mathrm{~m} \cdot \mathrm{s}^{-1}$ pour déclencher le transport de la neige et modifier fortement la répartition spatiale. Ce phénomène est à l'origine de la formation de suraccumulations qui peuvent atteindre plu- sieurs mètres d'épaisseur. On estime que $80 \%$ des avalanches majeures ont vu leur zone de départ modifiée par le transport de neige par le vent. Pour étudier ces avalanches que nous appellerons catastrophiques, il nous faut comprendre l'ensemble des processus qui contribuent à leur formation. En effet, pour disposer de la répartition de la neige dans la zone de départ d'un couloir, il est nécessaire d'avoir localement la précipitation de neige et le vent local. Nous avons donc besoin d'un moyen qui permet de déterminer le vent local à partir du vent moyen au niveau du massif et d'un outil de simulation du transport de la neige par le vent au voisinage immédiat de la zone de départ. Une fois le manteau neigeux en place, il nous faut comprendre et modéliser les processus de déclenchement et d'écoulements de ces avalanches. L'analyse des processus physiques qui gouvernent la formation et l'écoulement des avalanches catastrophiques et le développement des outils de modélisations associés sont l'objet de notre travail de recherches.

Ainsi, à partir de l'analyse des différentes étapes, c'est-àdire la formation du manteau (la superposition de couches de neige différentes), sa rupture et l'écoulement lui-même, nous espérons pouvoir simuler ces avalanches avec comme objectif la création d'un outil intégré pouvant simuler les avalanches majeures. Ces outils sont destinés à aider les collectivités locales à améliorer leurs plans d'occupation des sols et d'aménagement du territoire.

\subsection{Modélisation du transport de neige par le vent}

Une analyse de vingt grands événements d'avalanches majeures dans les Alpes françaises (Meffre, 1997) a montré que les conditions d'occurrence sont des fortes précipitations ventées par un temps froid. Le manteau neigeux relatif à ce 
type de situation est composé de deux importantes contributions : la précipitation et la neige transportée par le vent. La connaissance de la répartition spatiale de la neige dans la zone de départ doit intégrer ces deux composantes.

Dans la nature, la neige érodée sur le versant au vent est incorporée au flux d'air pour former un écoulement diphasique. Localement, derrière la crête, une zone de faible vitesse provoque la sédimentation des particules dans l'écoulement et leur dépôt. Il s'ensuit une suraccumulation de neige derrière la crête induisant une surcharge pondérale importante. Cette dernière est susceptible de provoquer la rupture d'une couche fragile enfouie dont nous examinerons plus loin comment les propriétés et le mode de fonctionnement ont été étudiés.

Un des moyens les plus anciens d'étude de la répartition spatiale de la neige sous l'effet du vent est la modélisation physique. Son principe est de reproduire sur modèle réduit, en soufflerie, un épisode de transport de neige. Le passage du modèle réduit à la réalité se fait par l'intermédiaire de critères de similitude. Ces derniers sont déterminés en examinant les équations de bilan écrites sans dimension. La complexité des phénomènes en jeu ne permet pas de respecter tous les critères de similitude identifiés, que les particules utilisées soient des particules sèches ou de la neige, d'où le développement ces dernières années de la modélisation numérique. L'étude des critères de similitude pour les écoulements diphasiques a fait l'objet d'une étude spécifique, grâce à diverses expérimentations menées in situ et dans la soufflerie du Cemagref. Dans le cas du transport de la neige par le vent, le modèle numérique développé au Cemagref et appelé NEMO, permet d'étudier le développement des deux couches de saltation et de diffusion turbulente responsables du transport. Il s'appuie sur les équations classiques de la mécanique des fluides en établissant un bilan de masse et de quantité de mouvement du fluide et des particules au sein de chaque maille du domaine avec introduction d'un flux d'érosion et de dépôt. Cette modélisation a reproduit avec succès les résultats d'expérimentations réalisées en soufflerie. Ce modèle est actuellement en cours de test pour des dépôts réels. En effet pour pouvoir calibrer les paramètres du modèle pour chaque type de neige et parvenir à une véritable validation, il est important de comparer le modèle à des observations in situ. Pour acquérir ces données, le Cemagref a équipé le site du Lac Blanc (domaine de l'Alpe d'Huez) en collaboration avec le Centre d'Etudes de la Neige (Météo France) et a réalisé des tests en soufflerie climatique du CSTB (avec de la neige artificielle).

\subsection{Modélisation de la stabilité du manteau neigeux}

Le but de cette modélisation est la caractérisation spatiale de la rupture d'une plaque. Le modèle doit pouvoir produire des scénarios de rupture conformes à ceux observés sur le terrain ou en laboratoire. S'agissant du terrain nous verrons dans quelle mesure le cadre d'étude des avalanches majeures nous permet de lever une certaine incertitude relative à la rhéologie du matériau neige à prendre en compte.

Avant d'examiner comment le modèle numérique de stabilité utilise les informations fournies par le modèle de transport de la neige par le vent, nous allons détailler les mécanismes physiques intervenant lors de la rupture d'une plaque.

La nature stratifiée du manteau neigeux est à la base de l'instabilité des couches de neiges qui provoquent les ava- lanches. En effet les différentes couches de neige, de par l'action du vent lors de leur formation et des transformations thermodynamiques qu'elles subissent par la suite, ont des propriétés mécaniques différentes. Ainsi un plan de glissement préférentiel est susceptible d'apparaître à l'interface entre deux couches de propriétés mécaniques différentes ou au sein d'une couche composée d'une neige de faible cohésion. Un cas particulier fait l'objet d'études au cemagref. Il s'agit de la couche dite fragile, composée de grains de neige appelés gobelets (type de neige se formant sous l'influence d'un fort gradient de température au sein de la couche). En effet les couches de gobelets sont caractérisées par un indice des vides élevé et par une cohésion extrêmement faible. Leur comportement est alors considéré comme étant rigide, fragile, ce qui leur confère une forte instabilité.

Lorsque de telles couches sont enfouies, elles sont susceptibles, sous l'influence de la surcharge des couches supérieures, de s'effondrer (ou de cisailler), ce qui génère l'apparition d'un plan de glissement en profondeur et la rupture en traction de la plaque.

\subsection{Le modèle numérique de stabilité}

D’une manière générale, il s'agit de code de calcul dynamique du type éléments ou différences finis permettant d'implémenter différentes lois de comportement classiques, de façon à calculer le champ de contrainte dans les deux couches et ainsi à déterminer la localisation spatiale de la rupture.

Pour fonctionner le modèle requière plusieurs données d'entrée :

- les caractéristiques mécaniques du matériau qui compose les différentes couches et leur loi de comportement,

- une loi d'interface décrivant le comportement de la couche fragile,

- une description géométrique de la structure étudiée,

- une description de la topographie de la pente sur laquelle repose le matériau étudié.

L'un des points les plus délicats de cette démarche numérique est le choix de la loi de comportement à prendre en compte pour décrire au mieux le matériau neige. Dans le cas général, les couches de neige étant soumises à des transformations thermodynamiques et mécaniques incessantes, le matériau voit sa loi de comportement évoluer au cours du temps. Il n'existe donc pas une neige et une loi de comportement pour cette neige mais plusieurs neiges et plusieurs lois de comportement.

Il est peu réaliste d'envisager de caractériser in situ, pour chaque cas envisagé, le comportement mécanique du matériau. Les problèmes de mesures liés à la fois à l'instrumentation et à la nature hétérogène du matériau (problème de représentativité de la mesure dans les matériaux inhomogènes) en sont responsables.

Une démarche plus adaptée aux contraintes imposées par le matériau neige et par le contexte dans lequel l'étude de sa stabilité intervient (contexte haute montagne) est donc nécessaire. Notre approche repose sur le contexte initial de notre étude : celui des avalanches majeures qui impliquent la rupture de plaques de neige récentes et n'ayant que peu évolué du point de vue thermodynamique et mécanique. Dans ce cas les propriétés mécaniques du matériau font état d'une faible dispersion, et une rhéologie moyenne peut être envisa- 
gée. Le matériau est alors caractérisé par une loi de comportement simple du type Mohr Coulomb. Il s'agit là d'une simplification de la réalité et nous verrons que les expérimentations de laboratoire, qui utilisent un matériau dont les propriétés moyennes sont proches de celle du matériau neige à prendre en compte dans le cadre des avalanches majeures, justifient cette approche. En effet nous verrons que dans certains cas (qui se trouvent être ceux qui sont le plus souvent observés sur le terrain) les caractéristiques topographiques et géométriques du problème prennent le pas sur la rhéologie du matériau et fixent la localisation spatiale de la rupture.

Le paramètre de sortie du modèle de stabilité est le volume de neige initial mis en mouvement dans la zone de départ connaissant la localisation spatiale de la rupture et l'épaisseur de la plaque. Ce paramètre est, comme nous allons le voir à présent, un des paramètres essentiels de la modélisation de l'écoulement des avalanches.

\subsection{Modélisation numérique de l'écoulement des avalanches}

Dans un premier temps, les écoulements denses et les aérosols étaient simulés par des modèles distincts dits de la dynamique du point, dans lesquels l'écoulement est un bloc indéformable pour les avalanches denses et déformable pour les avalanches aérosols (pour tenir compte de l'incorporation de l'air ambiant). Ces deux avalanches sont assimilées à un point dont on étudie la trajectoire.

Puis, dans les années 1980, des modèles plus complexes sont apparus : les avalanches étaient étudiées comme l'écoulement d'un fluide déformable dans le cadre de la théorie des milieux continus. Toutefois, la plupart des avalanches catastrophiques sont composées d'une partie dense en contact avec le sol et d'une partie aérosol qui recouvre la partie dense (Naaim, 1999). Les modèles existants étaient donc insuffisants et ne rendaient pas compte de la complexité de ces écoulements et tout particulièrement la formation d'un aérosol qui ne se produit qu'à la suite d'un processus complexe où l'interaction de l'avalanche dense avec l'air joue un rôle déterminant dans la formation et la croissance de l'aérosol comme le confirment les expériences effectuées sur le terrain en France, en Suisse, au Japon et en Norvège.

Nous avons alors développé un modèle pour les avalanches mixtes qui traite l'écoulement, du moment où il se déclenche jusqu'à son arrêt. La couche inférieure, de forte concentration volumique, se comporte comme un écoulement dense dont le principal paramètre est l'angle de frottement dynamique neige sur neige. Pour les avalanches catastrophiques on considère que la neige s'écoule sur de la neige en place. La simulation de cette couche se fonde sur les lois de conservation de la matière et de la quantité de mouvement complétées par des modèles issus de la théorie des milieux granulaires. Dans les équations des écoulements denses, on intègre des termes qui représentent les flux de masse et de quantité de mouvement échangés entre la phase dense et le manteau immobile et entre la phase dense et la phase aérosol. À mesure que l'aérosol progresse, son volume augmente, et le nuage de neige qui le compose s'étend par échange de neige avec la phase dense et par incorporation d'air. Nous supposons que la zone qui sépare les deux couches de l'écoulement se comporte comme une couche de saltation. Nous retrouvons dans les équations de cette zone les termes des flux de masse et de quantité de mouvement échangés : ils représentent l'érosion ou le dépôt des grains de neige. La quantité de neige prélevée est proportionnelle à la différence des contraintes exercées par les turbulences de l'aérosol et les forces de frottement inter-granulaires et de cohésion qui résistent à l'arrachement des particules dans la couche dense.

Nous avons défini les plages de variation des paramètres de ce modèle. Les valeurs de ces paramètres sont mesurées soit lors d'expériences en laboratoire, soit par des mesures effectuées sur le terrain.

\section{II — LA MODÉLISATION DE LA STABI- LITÉ EN LABORATOIRE}

Initialement ce sont les difficultés rencontrées sur le terrain pour caractériser mécaniquement le matériau neige impliqué dans la stabilité des zones de départ d'avalanches qui ont motivé notre choix de travailler en laboratoire.

Le manteau neigeux fait état, dans le cas général, d'une forte variabilité spatiale et temporelle. En effet, sous l'effet du transport éolien particulièrement actif dans les zones de départ d'avalanches, une même couche est susceptible de voir son épaisseur varier du simple au double en quelques mètres, de même que ces caractéristiques mécaniques. En outre, loin d'être homogène, le manteau neigeux contient de nombreuses inclusions de densités différentes constituant une distribution aléatoire d'hétérogénéité dans le milieu. On est alors confronté à un problème d'échantillonnage, le problème étant de déterminer un échantillon moyen représentatif de la pente. D'autre part, la neige étant en évolution thermodynamique permanente, et les variations influençant fortement les paramètres mécaniques, une mesure à un instant donné n'est valable qu'à cet instant. Comme nous pouvons le constater, l'étude de la stabilité de la neige est un problème multi-paramétré, et comme tout système complexe. le comportement paraît souvent chaotique. C'est pourquoi nous avons choisi de travailler en modèle réduit sur des matériaux modèles (poudre ou neige artificielle), plus homogènes, dont les propriétés mécaniques sont plus accessibles et plus facilement contrôlables. Le problème de la représentativité de la mesure se trouve alors en partie résolu. En outre, le nombre de paramètres impliqués dans la stabilité étant considérablement réduit, et chaque paramètre étant contrôlable par l'expérimentateur, il devient possible de faire varier un seul paramètre en laissant les autres constants, de façon à juger de son influence sur le comportement. Cette méthode de discrimination du rôle de chaque paramètre permet, entre autres, de mettre en évidence ceux d'entre eux qui, dans certaines situations déterminées, dominent le comportement et fixent la localisation spatiale de la rupture.

Les tests en laboratoire utilisant des poudres ont été réalisés au Cemagref de Grenoble. Il s'agit de reproduire, à l'échelle réduite des ruptures de "plaques" en utilisant une stratification bi-couche : une couche cohésive reposant sur une sous-couche fragile et bénéficiant d'un encrage amont. Les caractéristiques mécaniques des poudres composant les deux couches sont déterminées par le Cemagref de Bordeaux. La poudre cohésive, dérivée de la chaux, utilisée dans la couche supérieure, a une granulométrie moyenne de 40 microns et fait état d'une loi de comportement élastoplastique. La poudre utilisée pour simuler le comportement non 
cohésif des couches fragiles est un sable d'Hostun mono-disperse de granulométrie de 100 microns.

\subsection{Tests sur poudre en laboratoire}

Nous disposons d'un banc d'essais continûment inclinable, reproduisant une pente de géométrie simplifiée, puisqu'il s'agit d'une simple rupture de pente d'angle variable. La pente est recouverte d'une rugosité de taille compatible avec la taille de grains composant la couche inférieure. On dépose sur cette géométrie une couche fragile constituée de poudre non cohésive sur laquelle on dépose au tamis, de façon homogène, une couche de poudre cohésive. La plaque vient prendre appui sur un pied de butée aval et est retenue par un ancrage amont au niveau de la rupture de pente. De façon à éviter les effets de bords latéraux au contact entre la plaque supérieure et les bords transparents de canal, les bords latéraux de la couche supérieure reposent directement sur la rugosité. Ainsi seule la partie centrale de la plaque repose sur la couche fragile et est susceptible de se déstabiliser. Lors de la rupture, il apparaît donc une zone de cisaillement entre la poudre en mouvement et celle qui reste en place sur la rugosité. Le contact latéral est ainsi ramené à un cisaillement au sein même de la poudre cohésive, ce qui simplifiera la prise en compte de conditions aux limites latérales du problème.

Lorsque l'on augmente l'angle d'inclinaison de la pente, une rupture en cisaillement apparaît dans la couche fragile ; la plaque cohésive supérieure est alors soumise à une contrainte en traction au niveau de la rupture de pente, ce qui aboutit rapidement à sa rupture en traction. La rupture en cisaillement aux bords de la plaque et au pied de buté intervient rapidement après une phase de déformation plastique sans qu'il soit nécessaire d'augmenter l'angle une fois la rupture en traction apparue.

Ce dispositif expérimental est équipé d'une caméra vidéo filmant dans un plan fixe perpendiculaire à la pente, à raison de 25 images par seconde, et permettant de suivre image par image la chronologie de la rupture de façon à déterminer son point d'initiation ainsi que la façon dont elle se propage. La figure 1 illustre la naissance de la fissuration de la couche supérieure cohésive.

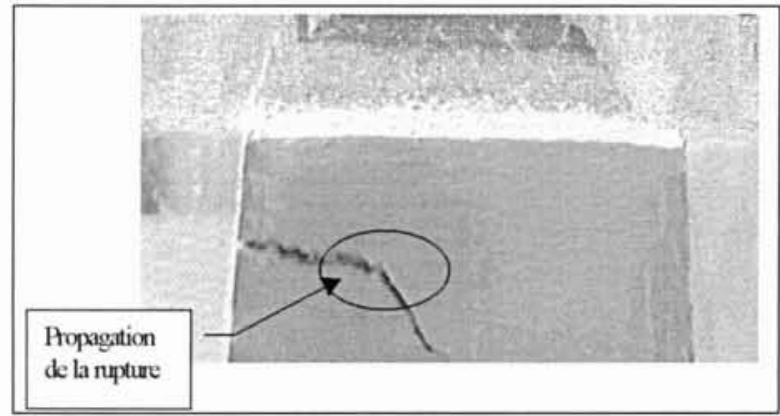

1. Simulation de rupture en mode couche fragile sur modèle réduit.

\footnotetext{
Chaque rupture de plaque est reliée aux paramètres physiques agissant sur la stabilité :

- Epaisseur et densité de la couche supérieure,

- Epaisseur de la couche fragile,

- Angle nécessaire pour obtenir la rupture qui fixe l'angle
} limite de stabilité de la pente.
De façon à assurer la reproductibilité des expériences, l'humidité relative de l'air était contrôlée.

Nous avons fait varier chacun des paramètres en laissant les autres constants de façon à déterminer le rôle joué par chaque paramètre indépendant dans la stabilité. Ainsi la stabilité, qui dans notre expérience se traduit par la valeur de l'angle limite de stabilité de la pente, peut être étudiée en fonction de l'épaisseur de la couche supérieure, de sa densité et de l'épaisseur de la couche fragile. Une étude de la localisation spatiale de la rupture a été réalisée pour différentes configurations géométriques (épaisseurs des deux couches), différentes densités de couche supérieure et différentes valeurs de la rupture de pente.

\section{III — RÉSULTATS DE L'ÉTUDE}

\subsection{La stabilité en fonction de l'épaisseur de la plaque supérieure}

L'une des premières questions qui se posait était de savoir si la chronologie de la rupture "rupture initiale en cisaillement dans la couche fragile suivie d'une rupture en traction dans la couche supérieure" était bien observable.

Une rupture en cisaillement dans la couche fragile apparait lorsque la contrainte de cisaillement appliquée sur cette couche par la couche supérieure atteint une valeur critique, ce qui se traduit par:

$$
\tau(\alpha)=\tau_{c}=\rho g e_{p} \sin \left(\alpha_{s}\right)
$$

où $\rho$ est la densité de la couche supérieure, $\alpha$ l'angle d'inclinaison de la pente par rapport à l'horizontale et $\alpha_{\mathrm{s}}$ la valeur de l'angle limite de stabilité de la pente. Cependant l'apparition d'une rupture en cisaillement dans la couche fragile n'est pas une condition suffisante pour atteindre la rupture de l'ensemble de la structure. En effet, après cette rupture initiale, une augmentation additionnelle de l'angle de $\Delta \alpha$ est nécessaire pour atteindre la rupture en traction de la plaque supérieure pour un angle correspondant égale à $\alpha_{p}$. On peut alors écrire :

$$
\alpha_{p}=\alpha_{s}+\Delta \alpha
$$

où $\alpha_{\mathrm{s}}$ est l'angle pour lequel apparaît la rupture en cisaillement de la couche fragile et où $\Delta \alpha$ est lié à la résistance en traction de la couche supérieure.

Si la couche supérieure n'était pas cohésive, la rupture globale de la structure bi-couche interviendrait dès la rupture en cisaillement de la couche fragile, c'est-à-dire lorsque :

$$
\tau(\alpha)=\tau_{c}=\rho g e_{p} \sin \left(\alpha_{p}\right)
$$

avec $\alpha_{\mathrm{s}}=\alpha_{\mathrm{p}}$;

ce qui peut être écrit :

$$
\sin \left(\alpha_{\mathrm{p}}\right)=\left(\tau_{\mathrm{c}} / \rho \mathrm{g}\right)\left(1 / \mathrm{e}_{\mathrm{p}}\right)
$$

Si l'on porte sur un graphique $\sin \left(\alpha_{\mathrm{p}}\right)$ en fonction de $1 / \mathrm{e}_{\mathrm{p}}$ on devrait donc observer une droite de pente $\tau_{\mathrm{c}} / \rho \mathrm{g}$.

Cependant la nature cohésive de la couche supérieure (et donc sa résistance en traction) conduit à prendre en compte 
un angle additionnel pour atteindre les conditions de rupture, ce qui conduit à écrire :

$$
\sin \left(\alpha_{\mathrm{p}}\right)=\left(\tau_{\mathrm{c}} / \rho g\right)\left(1 / \mathrm{e}_{\mathrm{p}}\right)+\mathrm{cte}
$$

Il est intéressant de noter qu'entre zéro et un certain seuil $1 / \mathrm{e}_{\mathrm{pw}}$ la courbe en pointillé n'a aucun sens physique.

En effet, lorsque l'épaisseur de la couche cohésive dépasse une valeur seuil $e_{p w}$, la rupture en cisaillement ne se fait plus dans la couche fragile mais directement dans la couche cohésive, le critère de Mohr Coulomb étant vérifié sur un plan appartenant à la couche supérieure. Dans ce cas, le mécanisme de rupture n'est plus conforme à celui pris en compte plus haut, ce qui graphiquement se traduit par une partie de la courbe non représentative à partir d'un certain seuil, correspondant à cette épaisseur limite.

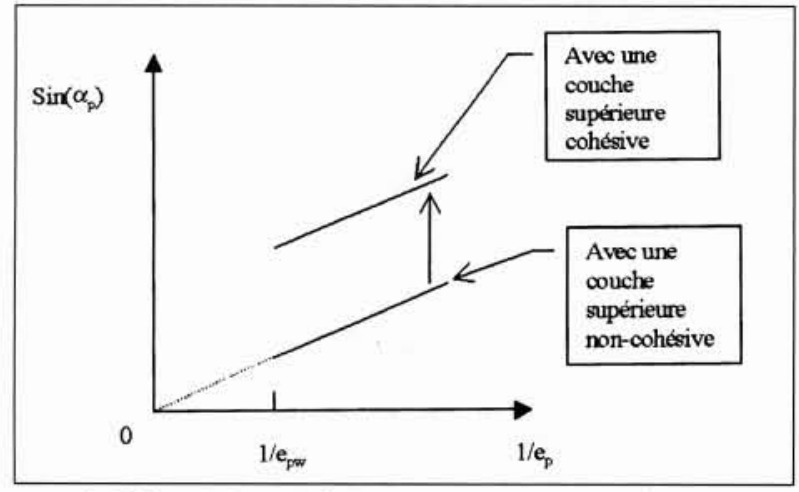

2. Effet de la cohésion de la couche supérieure sur la stabilité d'un système bicouche.

Nous avons réalisé, sur notre pente test, un ensemble de mesure pour des valeurs croissantes de l'épaisseur de la couche cohésive, en fixant sa densité ainsi que l'épaisseur de la couche fragile (qui était fixée à $8 \mathrm{~mm}$ ).

La densité moyenne de la couche supérieure était de 1,075 pour un écart-type de 0,02 . Cela confirme bien que la densité était fixée pour l'ensemble de ces expériences. Nous avons obtenu un bon accord avec la forme linéaire attendue (figure 3 ).

Dans la théorie de Mohr Coulomb la rupture en cisaillement dans la couche fragile apparaît lorsque le critère est satisfait, ce qui s'écrit classiquement :

$$
\tau=\tau_{c}=C+\sigma_{n c} \tan \left(\Phi_{w}\right)
$$

où $\mathrm{C}$ est la cohésion de la couche, $\sigma_{\mathrm{n}}$ la composante normale de la contrainte appliquée à la couche fragile et $\Phi_{w}$ son angle de frottement interne. Dans notre cas, nous avons fait l'hypothèse d'une cohésion nulle. Lorsque le critère est atteint nous pouvons écrire :

$$
\sin \left(\alpha_{\mathrm{s}}\right)=\left(\tau_{\mathrm{c}} / \rho \mathrm{g}\right)\left(1 / \mathrm{e}_{\mathrm{p}}\right), \text { c'est-à-dire } \alpha_{\mathrm{s}}=\Phi_{\mathrm{w} .}
$$

Le graphique 3 montre donc que l'utilisation du critère de Mohr Coulomb est bien appropriée à la description du comportement de la couche fragile.

Il montre de même que la chronologie de la rupture prise en compte pour notre étude est valide. En effet si la rupture avait lieu en premier dans la couche cohésive (deuxième type de mécanisme envisageable), l'angle correspondant à la rupture globale $\alpha_{p}$ serait indépendant de $e_{p}$. En effet pour des dimensions constantes de la plaque cohésive supérieure, la résistance en traction est indépendante de $\mathrm{e}_{\mathrm{p}}$.

J.C. Richards et R.L. Brown (1970) donnent l'expression suivante de la résistance en traction d'une couche cohésive :

$$
\mathrm{T}=(\mathrm{W} \sin \theta) / \mathrm{A}
$$

où $\mathrm{W}$ est le poids de la plaque en mouvement et $\mathrm{A}$ la surface de la zone de rupture et $\theta$ l'angle par rapport à l'horizontal lorsque la rupture en traction intervient.

Cette équation peut s'écrire :

$$
\mathrm{T}=(\rho \mathrm{LA} \sin \theta) / \mathrm{A}=\rho \mathrm{L} \sin \theta
$$

où $\rho$ est la densité de la plaque, $\mathrm{L}$ sa longueur et $\mathrm{A}$ la section de la zone de rupture, assimilée dans nos expériences à la section de la plaque. $\mathrm{Si}$, durant les expériences, les dimensions de la plaque ainsi que sa densité sont maintenues constantes, ce qui était le cas, on voit clairement que la résistance en traction de la plaque est, pour une certaine valeur de l'angle de rupture observé, une caractéristique intrinsèque de la plaque cohésive et ne dépend nullement de son épaisseur.

Dans l'hypothèse où la rupture prendrait initialement naissance dans la couche supérieure, formant ainsi une plaque qui glisserait sur la couche fragile en la cisaillant instantanément, l'angle de rupture globale de la pente $\alpha_{\mathrm{p}}$ serait identique à l'angle $\theta$ nécessaire pour obtenir la rupture en traction de la plaque. Celui-ci ne dépendant pas de $e_{p}$ aucune dépendance de la stabilité en fonction de l'épaisseur ne serait observable.

Or le graphique 3 montre clairement une dépendance de la stabilité en fonction de l'épaisseur de la couche cohésive, ce qui indique que la chronologie à prendre en compte est bien une rupture initiale dans la couche fragile suivie par une rupture en traction dans la couche supérieure et non l'inverse.

De plus il est bien conforme à ce que l'on peut attendre du fonctionnement "intuitif" d'une telle structure, à savoir que la pente est d'autant plus instable que l'épaisseur de la couche supérieure est importante.

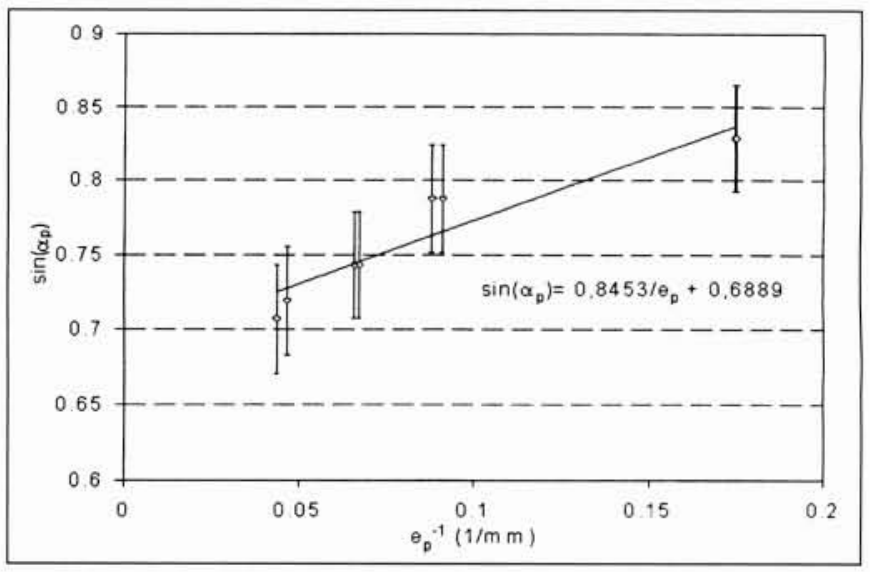

3. $\sin \left(\alpha_{\mathrm{p}}\right)$ en fonction de 1/ep. 

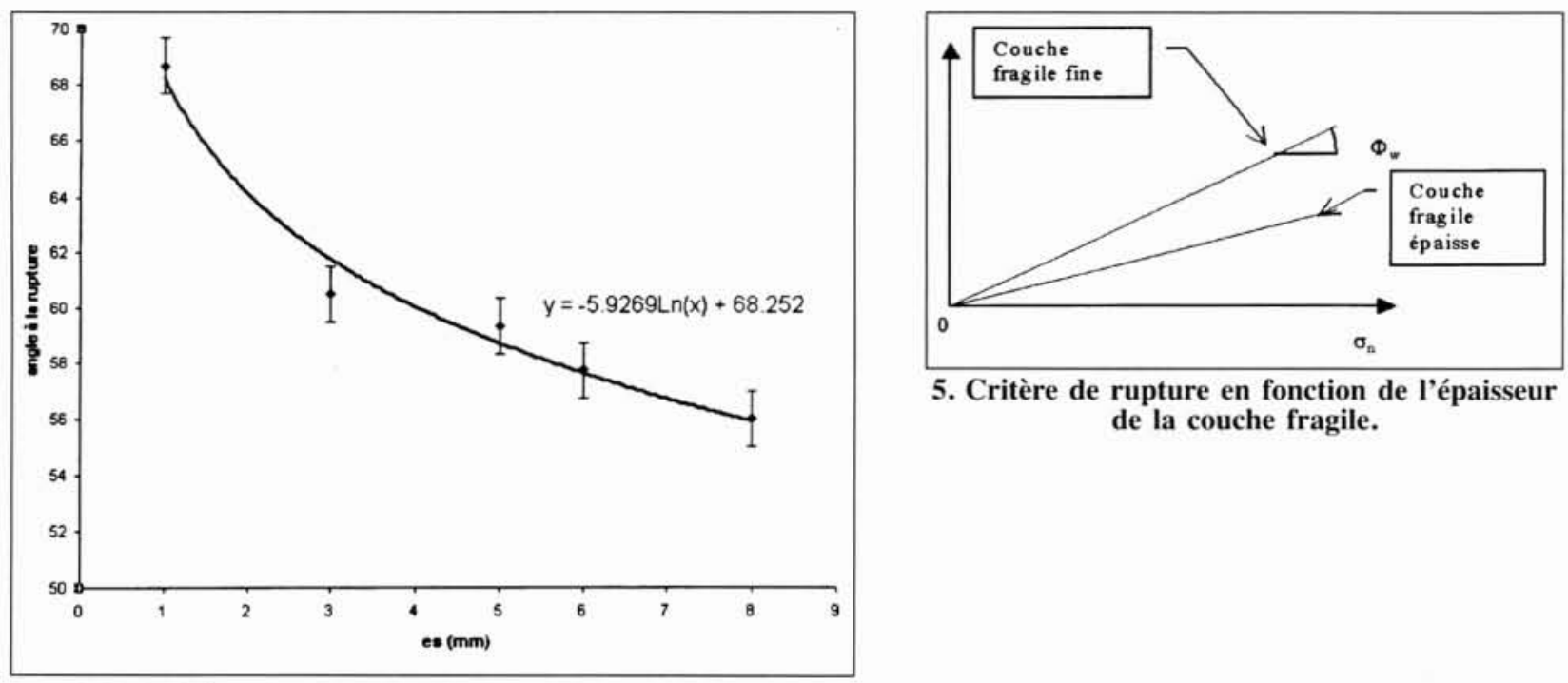

5. Critère de rupture en fonction de l'épaisseur de la couche fragile.

4. Stabilité en fonction de l'épaisseur de la couche fragile.

\section{- 3.2 La stabilité en fonction de l'épaisseur de la couche fragile}

La question qui se pose à présent est de déterminer le rôle de l'épaisseur de la couche fragile dans l'analyse de la stabilité. Pour répondre à cette question, nous avons cette fois travaillé à épaisseur et densité de couche supérieure constantes, en faisant varier l'épaisseur de la couche fragile.

L'épaisseur moyenne de la plaque supérieure était de 5,59 avec un écart-type de 0,4 .

La densité moyenne était de 1,16 pour un écart type de 0,065 . Les variations de $\alpha_{\mathrm{p}}$ en fonction de es sont portées sur la figure 4 . Ce graphique met en évidence une diminution de la stabilité lorsque l'épaisseur de la couche fragile augmente.

En effet, au bas de la couche, la rugosité, dont la taille caractéristique est compatible avec la taille des grains composant la couche fragile, assure un encastrement et donc une condition de non-glissement.

En haut de la couche, à l'interface en les deux couches, les grains composant la couche fragile et ceux beaucoup plus fins de la couche cohésive supérieure s'interpénètrent sur une épaisseur de quelques grains. Ainsi, la limite supérieure de la couche fragile se caractérise par une condition de non glissement. Cela a été vérifié en filmant l'expérience en mode macro focalisé sur l'interface entre les deux couches. ce qui a permis de constater l'apparition d'une bande de cisaillement, non pas à l'interface, mais en-dessous de celleci dans l'épaisseur de la couche fragile.

Ainsi les limites haute et basse de la couche se caractérisent par une forte résistance au cisaillement inter-granulaire se répercutant dans la structure granulaire voisine, sur une épaisseur de quelques grains, par transmission des forces de contacts par les grains considérés comme rigides (transmettant bien les contraintes). A une certaine distance de cette zone de contacts inter-granulaire non glissant (quelques diamètres de grains), la résistance au cisaillement inter-granulaire décroit et les grains, moins contraints, retrouvent un contact glissant.

Lorsque la couche fragile est assez épaisse, il existe entre les deux conditions aux limites une zone de contact glissant qui favorise l'apparition d'une bande de cisaillement conduisant à la rupture de la couche fragile. Cela se traduit, d'un point de vue macroscopique, par un angle de frottement interne relativement faible dans une bande de matériau au sein de la couche fragile.

Lorsque la couche est de faible épaisseur, les conditions de non glissement inter-granulaire se répercutent sur toute l'épaisseur de la couche, ce qui se traduit par une augmentation de l'angle de frottement interne de la couche.

Le critère est alors différent en fonction de l'épaisseur de la couche fragile considérée (figure 5).

D'autre part des expériences ont était réalisées, qui montrent qu'au-delà d'une certaine épaisseur de couche fragile la dépendance de la stabilité en fonction de l'épaisseur de la couche fragile n'est plus observée. En effet, à partir d'une certaine épaisseur, l'angle de frottement de la bande de matériau de contact inter-granulaire glissant tend vers l'angle de frottement interne du matériau non contraint (à surface libre) qui est la valeur minimum de l'angle de frottement du matériau. Dès lors, une augmentation de l'épaisseur de la couche fragile n'aura aucun effet sur l'angle de frottement interne de cette couche et donc aucun effet sur l'initiation de l'instabilité. La courbe 5 est donc bornée par cette limite supérieure.

\subsection{Stabilité en fonction de la densité}

De façon à déterminer l'influence d'une densité croissante sur la stabilité, des tests ont été réalisés en fixant l'épaisseur de la couche fragile à $8 \mathrm{~mm}$ et celle de la couche supérieure à $5,8 \mathrm{~mm}$ en moyenne sur l'ensemble des tests (avec un écart-type de 0,4$)$. La densité de la couche supérieure était quant à elle comprise entre 1,06 et $1,4 \mathrm{~g} / \mathrm{cm}^{3}$.

Théoriquement, une augmentation de la densité s'accompagne d'une augmentation du nombre de coordination, traduisant au niveau microscopique une augmentation des contacts inter-granulaires et au niveau macroscopique une augmentation de l'angle de frottement interne du matériau. D'autre part cette évolution du matériau s'accompagne d'une augmentation de sa résistance en traction, puisque celle-ci est directement reliée à la densité du matériau (pour des 
dimensions de plaques maintenues constantes et un angle d'inclinaison donné) par :

Ashton et al [1] donne une relation suivant une loi puissance reliant la résistance en traction à la densité par :

$$
\mathrm{T}=(\mathrm{cte})\left(\rho_{\mathrm{b}}\right)^{\mathrm{m}}
$$

Ainsi, on s'attend à une augmentation de l'angle de limite de stabilité de la pente, suivant une puissance de la densité lorsque celle-ci augmente (figure 6).

Notons que l'augmentation de la densité de couche supérieure s'accompagne aussi d'une augmentation de sa résistance au cisaillement, la cohésion et l'angle de frottement interne augmentant. Dans l'analyse de la stabilité, on pourrait a priori s'attendre à ce que la résistance en traction du matériau ne soit pas le seul paramètre à agir sur la limite de stabilité de la pente, mais que la

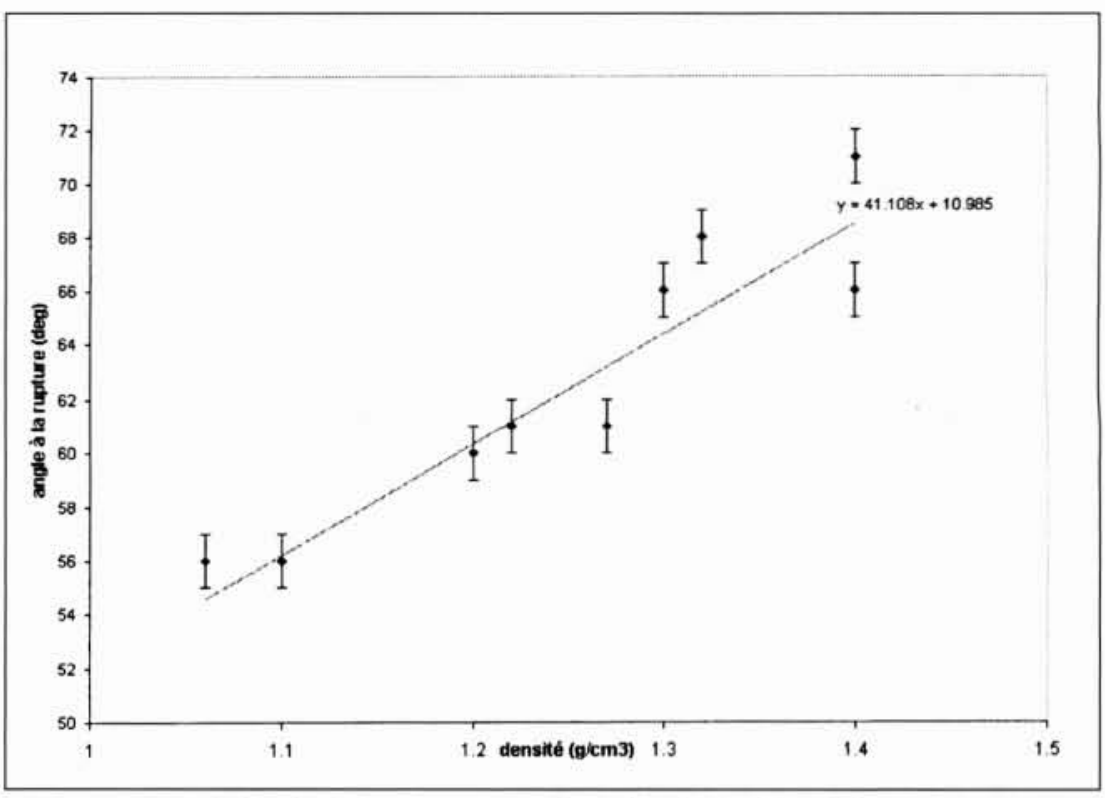

6. Angle à la rupture en fonction de la densité. résistance au cisaillement aux bords latéraux de la plaque supérieure ainsi qu'au niveau du pied de buté interviennent aussi. Cependant les ruptures en traction apparaissaient pour des angles d'inclinaison de la pente suffisamment importants pour que le critère de Mohr Coulomb (rupture en cisaillement) soit nécessairement vérifié aux bords latéraux de la plaque, ainsi que sur un plan à environ 45 degrés au niveau du pied de butée. Le critère de rupture de la plaque se confond donc ici avec le critère de rupture en traction de la plaque, ce qui justifie que, dans notre étude, la résistance en traction de la couche supérieure soit le seul paramètre pris en compte dans l'étude de la stabilité en fonction de la densité.

\subsection{Localisation de la rupture en traction en fonc- tion de la topographie}

Les films en vue de dessus de différentes expériences, réalisées en affectant des valeurs différentes à la rupture de pente amont, nous ont permis d'étudier la localisation spatiale de la rupture en traction de la plaque supérieure en fonction de la topographie (rupture de pente).

Pour les faibles valeurs de la rupture de pente (inférieure à 10 degrés), la localisation spatiale de l'initialisation de la rupture en traction se faisait de façon aléatoire, sans qu'il soit possible de la reproduire d'une expérience à l'autre. Au contraire, pour de fortes valeurs de la rupture de pente, la localisation spatiale de la rupture en traction était fortement déterministe, puisque cette dernière apparaissait systématiquement à la rupture de pente.

Deux effets antagonistes sont ici en compétition : le premier est lié, comme nous allons le voir, à la rhéologie du matériau. Le second est lié à la topographie de la pente.

Durant nos expériences, la couche supérieure était constituée de poudre cohésive fine dont les propriétés globales sont bien connues. Cependant à l'échelle micro structurale, les propriétés du matériau dépendent fortement du mode de préparation de la couche et de sa mise en place. En effet, des inclusions locales de densités différentes de la densité moyenne du matériau sont réparties de manière aléatoire lors

de la mise en place de la couche. Ces zones constituent des hétérogénéités, dont la distribution est aléatoire, et qui favorisent l'accumulation locale de la contrainte. La description du matériau en termes d'une loi de comportement globale, basée sur l'hypothèse d'un matériau homogène, n'est donc pas adaptée pour étudier la localisation spatiale d'une rupture en traction qui dépend essentiellement de la nature hétérogène du matériau. Lorsque la rupture de pente est faible, cet aspect hétérogène du matériau s'exprime pleinement. l'accumulation de contrainte se faisant majoritairement au niveau des hétérogénéités du matériau, puisque la zone de rupture de pente est peu sollicitée en traction. Au contraire, et c'est un cas particulièrement intéressant, lorsque la rupture de pente est forte, l'accumulation de contrainte est bien plus sensible au niveau de la rupture de pente qu'au niveau des hétérogénéités, ce qui a pour effet de localiser la zone de rupture à la rupture de pente. Tout se passe comme si, à forte rupture de pente, la topographie de la pente prenait le pas sur la rhéologie du matériau et fixait à elle seule la localisation spatiale de la rupture. Dans ce cas la description rhéologique globale du matériau, bien que ne tenant pas compte de la répartition des hétérogénéités, fixe la valeur de l'angle nécessaire pour atteindre la rupture en traction alors que sa localisation spatiale est fixée par la topographie.

L'intérêt majeur est alors que l'implémentation d'une loi de comportement simple (type Mohr Coulomb) nous permet, lorsque la topographie est assez marquée pour imposer la localisation de la rupture, de déterminer assez précisément le volume initial mis en jeu dans la zone de départ.

\section{- 3.5 Mécanisme de fonctionnement de la couche fragile}

A présent nous allons nous intéresser à la description microscopique du fonctionnement de la couche fragile et au mode de couplage entre les deux couches.

Dans un premier temps, de façon à déterminer le comportement réel de ces couches, des tests ont été réalisés in situ. La rupture de couche fragile était filmée à l'aide d'une caméra numérique focalisée en mode macro sur la couche 
fragile. La résolution était suffisante pour visualiser la couche à l'échelle du grain.

L'analyse des images ainsi obtenues à montré que :

- la perte de structure de la couche fragile implique au niveau microscopique un changement brutal des caractéristiques des liens inter-granulaires,

- au niveau macroscopique cette perte de structure se traduit par l'apparition d'une bande de cisaillement fortement contractant,

- au niveau microscopique, les grains (gobelets) sont liés par de petits ponts de glace, il s'agit d'un faible frittage. Cependant la nature fortement anguleuse de ces grains réduit fortement les points de contacts inter-granulaires, et donc le nombre de ponts de glace entre les grains. La cohésion de frittage est donc faible, mais néanmoins présente. Le comportement mécanique des liens inter-granulaires peut être, en première approximation, assimilé à un lien rigide fragile : ainsi, à partir d'un certain seuil de contrainte, ces liens sont susceptibles de se briser. Le matériau passe alors d'un état faiblement cohésif à un état totalement non cohésif. Nous avons donc qualifié le comportement de ces couches fragile de faiblement cohésif, à cohésion évanescente.

De plus, la faible cohésion de frittage permet au matériau de se maintenir dans une configuration de fort indice des vides fortement instables, les liens rigides empêchant les grains de réduire leur énergie potentielle en évoluant vers une configuration de faible indice des vides, naturellement plus stable. La couche a donc, dans cet état, une forte énergie potentielle et donc un fort potentiel d'instabilité. Lorsque, sous l'effet de la charge de la plaque supérieure, le seuil de contrainte est atteint et que les liens se brisent, l'énergie potentielle du système diminue de façon critique. ce qui se traduit, du point de vue de la structure, par une forte diminution de l'indice des vides.

\subsection{Elaboration du matériau en laboratoire}

De façon à reproduire le comportement décrit ci-dessus, le phénomène de foisonnement dans le sable a été utilisé : on incorpore dans le sable une quantité d'eau correspondant à $10 \%$ de la masse sèche du sable. Cette eau est répartie de façon homogène dans l'ensemble du réseau poreux par brassage. Le résultat est l'apparition d'un film capillaire entre les grains qui confère à la structure une légère cohésion capillaire. Cette dernière est suffisante pour permettre aux grains de se réarranger en créant des voûtes au sein même de la structure, ce qui a pour effet de former des vides. Du point de vue de l'indice des vides cette solution est tout à fait satisfaisante ; cependant elle implique une cohésion capillaire entre les grains et donc un lien visqueux incompatible avec les liens rigides fragiles que nous cherchons à modéliser. Il nous faut donc éliminer la phase liquide tout en conservant la structure de fort indice des vides. Cela est rendu possible par l'ajout d'un liant (type laque) dans l'eau que l'on incorpore au sable. Le mélange ainsi obtenu est un sable humide, de fort indice de vide, et dont les liens intergranulaires sont composés d'un ménisque liquide d'eau et de particule de laque. La couche fragile est alors mise en place sur le canal expérimental, dans cet état humide, puis elle est recouverte de la couche supérieure. Une fois la structure mise en place, on soumet l'ensemble à un fort rayonnement infrarouge qui assèche la couche fragile. L'eau présente entre les grains s'évapore alors que la laque forme un pont solide entre les grains. Le lien ainsi formé est extrêmement fragile et répond donc bien aux exigences que nous avions définies.

Le matériau ainsi obtenu a un indice des vides élevé ainsi qu'une micro-cohésion qui disparaît lors de très faibles sollicitations extérieures, ce qui confère à l'ensemble une structure fragile particulièrement instable.

Ce matériau a été utilisé pour étudier les différents comportements de couches de matériau granulaire fragile. Plus particulièrement le couplage entre la couche fragile et la couche supérieure cohésive a été étudié ainsi que les différents modes de fonctionnement du système bicouche qui en découle.

Dans cette étude, un modèle de plaque a été employé pour reproduire le comportement d'une plaque plus ou moins déformable. Il s'agit d'une succession de petits blocs rigides reliés entre eux par des liens élastiques de raideur plus ou moins grande. D'après les expérimentations que nous avons menées, deux types de fonctionnement de la couche fragile, liés aux propriétés mécaniques de la couche supérieure d'une part et au mode de sollicitation (naturelle ou provoquée) de l'autre, semblent devoir être pris en compte.

\subsection{Cas de mécanismes de rupture naturels}

L'accumulation de neige, liée aux précipitations et au transport de neige par le vent, induit une surcharge pondérale que la plaque répartit sur l'ensemble de la couche fragile sous-jacente. Cela est rendu possible par le fait que la plaque, composée de neige fortement frittée, fait état d'une forte rigidité et permet donc une bonne répartition latérale de la charge. Lorsque la contrainte de cisaillement atteint sa valeur critique dans la couche fragile, il apparaît un plan de cisaillement dans cette couche qui mène à son effondrement. Il s'agit là d'un phénomène de localisation de la déformation dans la couche fragile sur une bande de cisaillement. Les forces de frottement sous la plaque s'annulent alors brutalement induisant une réorganisation brutale des conditions aux limites en contrainte de la plaque, ce qui aboutit à sa rupture en traction. Il s'agit d'un mécanisme global impliquant l'effondrement de l'ensemble de la couche fragile au même instant, par opposition au cas d'un effondrement local se propageant à l'ensemble de la couche.

En laboratoire, ce processus est modélisé de la façon suivante :

- la plaque supérieure est constituée de blocs rigides reliés par des liens de forte raideur conférant à la plaque ainsi formée une rigidité importante. Cette plaque repose sur une couche fragile très faiblement cohésive et de fort indice des vides. L'augmentation de la pente sur laquelle repose cette structure provoque une augmentation de la contrainte globalement exercée sur la couche fragile et l'apparition d'une bande de cisaillement globale, concernant l'ensemble de la couche fragile au même instant.

\subsection{Mécanisme de départ accidentel}

Dans ce cas il s'agit d'une perturbation locale de la couche fragile par une surcharge instantanée appliquée à la couche supérieure. Localement sous la zone de surcharge, la couche fragile s'effondre, ce qui se traduit par une forte diminution de son épaisseur. Cela induit une déformation verticale de la plaque supérieure qui s'adapte localement à la nouvelle géométrie de la couche fragile sur laquelle elle repose. Cette déformation locale se propage alors dans la 
plaque supérieure, et induit de proche en proche l'effondrement de toute la couche fragile. Notons que cette propagation est d'autant plus observable que la plaque supérieure est déformable.

En laboratoire, ce processus est modélisé de la façon suivante :

- la plaque supérieure est, cette fois, constituée de blocs rigides reliés par des liens élastiques de faible raideur conférant à la plaque ainsi formée une forte capacité à se déformer. Cette plaque repose, comme précédemment, sur une couche fragile très faiblement cohésive et de fort indice des vides.

Un impact local est provoqué par la chute d'une masse sur le premier bloc en aval de la pente. Le processus est filmé en vue latérale à travers une vitre. On observe alors une propagation de proche en proche du déplacement des blocs de la couche supérieure couplée à un effondrement de la couche fragile sur laquelle reposent les blocs.

\section{IV — CONCLUSION}

Nous avons vu comment la modélisation de la stabilité trouvait sa place dans une chaîne de modèles imbriqués allant de l'étude du transport de la neige par le vent et de son influence sur la répartition de la neige dans les zones de départ des avalanches, au modèle d'écoulement permettant de déterminer la zone d'influence d'une avalanche.

En outre, la modélisation de la stabilité en laboratoire a permis de déterminer les typologies pour lesquelles une analyse simple de paramètres accessibles peut apporter de l'information quant au volume initial mis en jeu dans la zone de départ.

D'autre part, une analyse micro-structurale de la couche fragile a permis de dégager deux types de mode de fonctionnement du système plaque cohésive - couche fragile, qui pourront nous aider à mieux appréhender la complexité du phénomène in situ.

Des modélisations numériques visant à reproduire les mêmes phénomènes que ceux observés en laboratoire, tant à l'échelle macroscopique par l'intermédiaire d'une modélisa- tion aux différences finies qu'à l'échelle microscopique par le biais d'une modélisation aux éléments distinct, sont en cours.

Enfin une fois l'approche numérique validée sur les tests en laboratoire, elle sera appliquée à des cas de terrain simples et bien décrits de façon à aller vers un objectif opérationnel par l'utilisation des modèles de transport, de stabilité et d'écoulement dans un système intégré d'aide à la décision.

\section{BIBLIOGRAPHIE}

[1] Ashton, M.D. Cheng, D.C.H. Faraley, R and VAl.ANTiN. F.H.H.(1965), Some investigations into the strength and flow properties of powders. Gheologica Acta 4. (Oct.). 206-17

[2] Brown, R.L. and Richards, J.C. Principles of powder mechanics, Pergamon press (1970)

[3] Ching S.Chang (1993), Dislocation and plasticity of granular material with frictional contacts, powders and grains 1993, 105-110.

[4] Jamieson BrucE, (1999), Skier triggering of slab avalanches: Concepts and research, The avalanche review, vol 18. No 2 December 1999.

[5] Jean, M., Radjal, F. and Wolf, D.E., Roux, S., Moreau, J J. . (1997), Force network in dense granular media. Powders and grains 1997, 211. 214.

[6] LANG R.M., HARRISON, W.L 1995, Triaxial tests on dry, naturally occurring snow, Cold Region Science and Technology 23, 191-199

[7] McCung D.M, Jurg SChweizer, (1999) Skier triggering, snow temperature and the stability index for dry-slab avalanches initiation. Journal of glaciology Vol 45, 190-200.

[8] Meshike, G., LiU, C. and MANg, A. (1996). Large strain finite element analysis of snow. Journal of engineering mechanics 122, 591-602

[9] MiRSA, A. (1991), A micromechanics model for consolidated granular solids. Powders and grains 1993, 123-128.

[10] Richards, J.C. (1963), Shear and tensile strength measurements on powders exhibiting mild cohesion. Brit.Coal. Util. res. Assoc. Members' Private information Circular No 274

[11] Roscoe, K.H., SChofle.D, A.N, and Wroth. C.P. (1958), On the yielding of soils, geotechnique $8,22-53$.

[12] Yамmamoto, S, and Matsuoka, H. (1997). The Stress behavior of a granular material under shearing based on the mechanism of fabric change, Powders and Grains 1997, 195-198. 